\title{
Analysis and Research on Influencing Factors of Robust Operation of Community Elderly Canteen Based on ISM
}

\author{
Liu Changgui ${ }^{1, a}$, Chen Liang $^{2, b^{*}}$ \\ ${ }^{1}$ School of Design, South China University of Technology, Guangzhou, Guangdong Province, China \\ ${ }^{2}$ School of Design, South China University of Technology, Guangzhou, Guangdong Province, China \\ a787859062@qq.com \\ b*29397914@qq.com
}

\begin{abstract}
In order to cope with the development of the aging society, the food service provided by Guangzhou for the elderly is the standard configuration of home care services in the current environment, and community elders canteens are an important part of the construction of care service facilities. On the basis of summarizing the influencing factors of the stable operation of the community elderly canteen in Guangzhou, after consulting with experts and on-site investigation, the internal and external factors affecting the stable operation of the elderly canteen were clarified, including eight factors. Use the ISM interpretation structure model to divide the factors that affect the stable operation of the elderly canteen in the community. The research shows that the market environment, policy environment, and social environment are the root factors that affect the stable operation of the elderly canteen. Human resources and technical capabilities are the intermediate factors that affect the stable operation of the elderly cafeteria, and service capabilities are the direct factors that affect the stable operation of the elderly cafeteria. Finally, based on this, it provides suggestions and directions for the decision-making of the elderly canteen.
\end{abstract}

Keywords: elder canteen, pension, operation, internal and external factors, ISM model

\section{基于 ISM 模型的社区长者食堂稳健运营影响因素分析 及研究 \\ 刘昌贵 $1, \mathrm{a}$, 陈亮 $2, \mathrm{~b}^{*}$}

1 华南理工大学设计学院, 广州, 广东省, 中国

2 华南理工大学设计学院, 广州, 广东省, 中国

a787859062@qq.com

b*29397914@qq.com

\section{摘要}

为应对老龄化社会趋势下的发展，广州市所展开的应对老年群体的助餐配餐服务是当前环境下居家养 老服务的标准配置, 社区长者食堂是养老服务设施建设中的重要部分。在总结广州市社区长者食堂稳 定运行影响因素的基础上, 在与专家咨询和现场调查后, 明确了影响老年食堂稳定运行的内部因素和 外部因素, 其中包括八个要素。使用 ISM 解释结构模型, 将影响社区长者食堂稳健运营的因素进行 层级划分, 经研究表明市场环境、政策环境、社会环境是影响长者食堂稳健运营的根源因素, 行业文 化、管理者思想先进程度、人力资源和技术能力是影响长者食堂稳健运营的中间因素, 服务能力是影 响长者食堂稳健运营的直接因素。最后据此为长者食堂的运营决策提供建议和方向。

关键词: 长者食堂、养老、运营、内外部影响因素、ISM 模型 


\section{4 理论综述}

\section{1.绪论}

\section{1 养老模式现状}

根据 2017 年国务院发布的《"十三五"国家老龄化事 业发展和养老体系建设规划》 ${ }^{\mathbf{I}} 1$, 中国将以家庭和社区 为基础, 辅以机构, 并与医疗系统相结合。按照这类养 老服务模式，家庭护理是以家庭为根本、为基础的，因 此普通住宅区是中国老年人的首要养老区域。这种模式 还需要社区护理服务网络的支持和国家系统政策法律 管理的保障。家庭养老模式所需的家庭服务是老年人是 否可以在自己的家中度过老年的关键。

广州市养老服务发展计划是"9064"，即 90\%的老人 住在社区家中， $6 \%$ 的老人通过政府购买社区老人护理 服务, $4 \%$ 的老人在老年护理服务中会集养老 ${ }^{\mathbf{I}_{2} \mathbf{1}}$ 。对于 有老年人的家庭，空巢老人占其中的 $40 \%$ 以上。除了残 疾或生病的老人以及需要他人照顾的老人外, 吃饭和用 餐已成为老人在家中能够照顾自己的居家养老的首要 需求。

\section{2 广州长者食堂发展现状}

从 2016 年 11 月至来, 广州市资助 3.88 亿元支持社 区展开家居护理服务。截至目前，全市已建成 144 个综 合家庭护理服务平台, 1144 个社区老年人活动场所, 475 个食品辅助点。社区的服务重点也是居家老人的吃饭问 题。因此, 助餐配餐服务也是未来广州居家养老服务的 标配。

2018 年 2 月, 全广州市区的长者食堂有 928 个，这 是根据地区的老年人数量和居住范围合理设定的一系 列数量。老年食堂的服务模式已形成社会化集中分布, 社区商店和食品辅助餐, 养老机构辐射服务, 食品服务 机构委托经营等服务模式, 设立 475 个食品援助点, 覆 盖 157 街（镇），1,422 社区（村）有 108 万老年人，其 中 8 个区完全覆盖了街道（镇）。

\section{3 研究目的和意义}

在过去, 随着城市里中高龄老人数量的不断增加, 一些独居老人、孤独老人和患病老人日常的就餐问题日 渐突出。长者食堂的建立是家庭护理服务的创新措施。 为老人提供每天的餐食, 让老人们可以很容易地吃到热 气腾腾的饭, 有着重要的可解决老龄化与空巢化叠加的 养老意义。可见长者食堂的出现是一件惠民利民的大好 事, 而纵观大部分食堂, 目前面对的问题是如何把长者 食堂持续开办。研究长者食堂的运营模式等因素进行分 析, 得出影响长者食堂稳健运营的因素, 并且给出科学 的观点, 为长者食堂能持续地开展下去, 有效提升老年 人的获得感和幸福感做出贡献。
在现有针对长者食堂类型体系或企业高效运营分 析的研究中, 李莉等人广州市社区长者食堂的空间调研 为基础，从长者食堂的建设模式、服务内容、内部空间 的使用情况等方面进行了分析，从系统和空间策略的角 度总结了老年食堂的建设经验 ${ }^{\mathbf{I}} \mathbf{1}$ 。左志平等人运用 SPSS19.0 采用主成分分析法分析了四组养猪户绿色运 行方式的影响因素, 最后获得了当今养猪户绿色运营模 式的驱动成分是政府的生态补助鼓励政策等结论, 并且 得出几个制约成分, 提出相关的对策和建议 ${ }^{4} \mathbf{I}$ 。杨宝龙 运用霍尔三维结构模型方法构建智能社区运营风险的 三维模型, 主要从因子维度、时间维度和目标维度来分 析社区运营风险之间的内在联系和影响，推导出了用户 风险对运营系统的风险值影响程度是最大的 ${ }^{\mathrm{I}_{5}}{ }^{1}$ 。

整合上述研究, 虽然上述文献为长者食堂的稳健影 响因素的评价工作的开展提供了部分有价值的价值参 考, 但现有的有关长者食堂方面的研究还是较少, 侧重 于食堂的空间形式。从其他方面看，除了食堂的空间形 式外, 还有更多的不同因素影响长者食堂的建设和稳健 经营。

\section{2.长者食堂高效运营影响因素的确定}

开设长者食堂的政策出台以来, 各街道的长者食堂 数量有所增加, 大部分有就餐需求的老年人都用上了餐 食。现有长者食堂的配餐服务一般主要是分为两种: 老 人预约餐食搭配并亲自到现场进餐和社工为他们亲自 送餐，选择第一种的老人基本是腿脚方便的老人，而第 二种针对的老年人群体是残疾老人、病种老人等，服务 比较完善。由于长者食堂针对的群体主要是老年人, 在 价钱的收取方面因此也是仅仅在成本价之上，其主要的 经济来源是政府的补助和社会的公益捐献, 因此想要维 持长者食堂本身的稳健运营是一个比较重要以及困难 的问题。针对影响长者食堂稳健运营的几个要素采取归 纳分析, 帮助提高长者食堂稳健的发展, 降低倒闭的风 险，持续满足老年人的饮食需求和提升老年人的就餐体 验, 进一步落实居家养老模式的宗旨。

通过对长者食堂的运营流程的分析和对长者食堂 的实地调研, 以及相关文献资料的整理归纳, 并结合有 关专家意见, 本文从长者食堂的内部因素和外部因素两 个方面篮选出 8 个影响长者食堂文稳健运营的因子, 有 人力资源、技术能力、服务能力、行业文化、管理者思 想先进程度、市场环境、政策环境、社会环境八个因素， 如表 1 。 
不存在影响; “X”表明行因素与列因素存在互相影响的 关系; “ $\mathrm{O}$ ”表明行列彼此之间是不存在影响 ${ }^{\mathbf{1}}{ }^{10} \mathbf{1}$ 。每个因 素的结构之间形成相互作用矩阵 (SSIM) 如表 2 所示。

表 2 各因素结构自相互作用矩阵

表 1 长者食堂运营影响因素

\begin{tabular}{|c|c|c|c|}
\hline 划分 & 因素 & 符号 & 内容/含义 \\
\hline \multirow{5}{*}{ 内部 } & 人力资源 & $\mathrm{S}_{1}$ & $\begin{array}{c}\text { 工作人员情况、人数、年 } \\
\text { 龄段 }\end{array}$ \\
\hline & 技术能力 & $\mathrm{S}_{2}$ & $\begin{array}{c}\text { 厨师对饭菜色香味以及软 } \\
\text { 硬口感的把控 }\end{array}$ \\
\hline & 服务能力 & $\mathrm{S}_{3}$ & $\begin{array}{c}\text { 内部现有服务的类型、服 } \\
\text { 务质量 }\end{array}$ \\
\hline & 行业文化 & $\mathrm{S}_{4}$ & 成熟的经营体系、宗旨 \\
\hline & $\begin{array}{c}\text { 管理者思 } \\
\text { 想先进程 } \\
\text { 度 }\end{array}$ & $\mathrm{S}_{5}$ & $\begin{array}{c}\text { 全心为老年人服务、经营 } \\
\text { 的经验 }\end{array}$ \\
\hline \multirow{3}{*}{ 外部 } & 市场环境 & $\mathrm{S}_{6}$ & $\begin{array}{c}\text { 物价水平 (场地租金、饭 } \\
\text { 菜材料等成本) }\end{array}$ \\
\hline & 政策环境 & $\mathrm{S}_{7}$ & 政府的条例以及资金支持 \\
\hline & 社会环境 & $\mathrm{S}_{8}$ & 社会捐助、企业扶持等 \\
\hline
\end{tabular}

\section{3.长者食堂稳健运营影响因素的 ISM 模型构 建}

\section{1 方法介绍}

ISM(Interpretation Structure Model) 分析方法由 John N. Warfield ${ }^{{ }^{\mathbf{I}} \mathbf{I}^{\mathbf{I}}}$ 在系统论的基础上起先提出, 是体系的工 程结构布局建模技术中最经常使用的技术, 它是用来定 性描述体系组成要素及要素间条理干系的解释模型。所 述 ISM 方式是利用关联矩阵方式来标出系统要素间便 于辨认的因果联系和不容易辨析的耦合干系 ${ }^{\mathbf{l} 7 \mathbf{1}}$, 使用 $\mathrm{n}$ 阶二元矩阵最多 $\mathrm{n}$ 次自乘布尔运算的结果趋于稳定的特 性, 利用数理逻辑推导的途径来解释复杂的层级结构 ${ }^{\mathbf{8}} \mathbf{1}$ 。 近年来, ISM 分析方法已被广泛应用在农业经济和工程 质量等方面, 如区域经济发展, 安全生产, 企业和经济 管理等 ${ }^{9}$ 】。

\section{2 建立相互作用矩阵}

本文用 $\mathrm{S}_{1} 、 \mathrm{~S}_{2} 、 \mathrm{~S}_{3} 、 \mathrm{~S}_{4} 、 \mathrm{~S}_{5} 、 \mathrm{~S}_{6} 、 \mathrm{~S}_{7} 、 \mathrm{~S}_{8}$ 分别表示: 人力资源、技术能力、服务能力、行业文化、管理者思 想先进程度、市场环境、政策环境、社会环境因素间的 逻辑关系。在文献归纳总结, 并咨询有关长者食堂的管 理专家以及学者的基础上给出上述 8 个因素间的逻辑关 系。此中, “V”表明行因素是对列因素存在直接抑或间接 的影响, 列因素对行因素是不存在影响; “A”表明列因素 是对行因素存在直接抑或间接的影响, 行因素对列因素

\begin{tabular}{|c|c|c|c|c|c|c|c|c|}
\hline 序号 & 致因因素 & $\mathrm{S}_{8}$ & $\mathrm{~S}_{7}$ & $\mathrm{~S}_{6}$ & $\mathrm{~S}_{5}$ & $\mathrm{~S}_{4}$ & $\mathrm{~S}_{3}$ & $\mathrm{~S}_{2}$ \\
\hline $\mathrm{S}_{1}$ & 人力资源 & $\mathrm{A}$ & $\mathrm{O}$ & $\mathrm{O}$ & $\mathrm{A}$ & $\mathrm{O}$ & $\mathrm{V}$ & $\mathrm{V}$ \\
\hline $\mathrm{S}_{2}$ & 技术能力 & $\mathrm{O}$ & $\mathrm{O}$ & $\mathrm{O}$ & $\mathrm{O}$ & $\mathrm{O}$ & $\mathrm{V}$ & \\
\hline $\mathrm{S}_{3}$ & 服务能力 & $\mathrm{A}$ & $\mathrm{O}$ & $\mathrm{A}$ & $\mathrm{A}$ & $\mathrm{A}$ & & \\
\hline $\mathrm{S}_{4}$ & 行业文化 & $\mathrm{A}$ & $\mathrm{O}$ & $\mathrm{O}$ & $\mathrm{V}$ & & & \\
\hline $\mathrm{S}_{5}$ & $\begin{array}{c}\text { 管理者思想 } \\
\text { 先进程度 }\end{array}$ & $\mathrm{O}$ & $\mathrm{O}$ & $\mathrm{O}$ & & & & \\
\hline $\mathrm{S}_{6}$ & 市场环境 & $\mathrm{O}$ & $\mathrm{O}$ & & & & & \\
\hline $\mathrm{S}_{7}$ & 政策环境 & $\mathrm{X}$ & & & & & & \\
\hline $\mathrm{S}_{8}$ & 社会环境 & & & & & & & \\
\hline
\end{tabular}

\section{3 建立邻接矩阵并生成可达矩阵}

根据社区长者食堂稳健运营影响因素关系表（见表 1) 和相互作用矩阵 (见表 2), 建立邻接矩阵 A, 用于 表明社区长者食堂稳健运营各影响因素之间的关联性。 然后确定随便两个元素 $S_{i}$ 与 $S_{j}$ 之间的直接影响关系, 邻接矩阵阐述了点之间的每个通过长度为 1 的通路互 相可以达到的情况, 当 $S_{i j}=1$ 时, 表示 $S_{i}$ 对 $S_{j}$ 有直接 影响或者影响很强; 当 $S_{i j}=0$ 时, 表示 $S_{i}$ 对 $S_{j}$ 没有影 响或者影响很弱 $\mathbf{I}_{11}$ 。

\section{表 3 要素邻接矩阵 A}

\begin{tabular}{|c|c|c|c|c|c|c|c|c|}
\hline & $\mathrm{S}_{1}$ & $\mathrm{~S}_{2}$ & $\mathrm{~S}_{3}$ & $\mathrm{~S}_{4}$ & $\mathrm{~S}_{5}$ & $\mathrm{~S}_{6}$ & $\mathrm{~S}_{7}$ & $\mathrm{~S}_{8}$ \\
\hline $\mathrm{S}_{1}$ & 1 & 1 & 1 & 0 & 0 & 0 & 0 & 0 \\
\hline $\mathrm{S}_{2}$ & 0 & 1 & 1 & 0 & 0 & 0 & 0 & 0 \\
\hline $\mathrm{S}_{3}$ & 0 & 0 & 1 & 0 & 0 & 0 & 0 & 0 \\
\hline $\mathrm{S}_{4}$ & 0 & 0 & 1 & 1 & 1 & 0 & 0 & 0 \\
\hline $\mathrm{S}_{5}$ & 1 & 0 & 1 & 1 & 1 & 0 & 0 & 0 \\
\hline $\mathrm{S}_{6}$ & 0 & 0 & 1 & 0 & 0 & 1 & 0 & 0 \\
\hline $\mathrm{S}_{7}$ & 0 & 0 & 0 & 0 & 0 & 0 & 1 & 1 \\
\hline $\mathrm{S}_{8}$ & 1 & 0 & 1 & 1 & 0 & 0 & 1 & 1 \\
\hline
\end{tabular}

按照可达矩阵公式 $\mathrm{M}=(\mathrm{A}+\mathrm{I}) \mathrm{r}=(\mathrm{A}+\mathrm{I}) \mathrm{r}+1(\mathrm{r} \geq 1$, $r \in z$ ), 其中 $I$ 是单位矩阵。可达矩阵一般是用于表示按 照一定的逻辑机理, 社区长者食堂稳健运营影响因素之 间经过一定长度的通路后可达到的程度。按照公式, 由 表 3 的计算可以得到可达矩阵 R，如下表 4: 
高要素为 $\mathrm{S}_{\mathrm{i}}$ 。根据 $\mathrm{R}\left(\mathrm{S}_{\mathrm{i}}\right) \cap \mathrm{Q}\left(\mathrm{S}_{\mathrm{i}}\right)=\mathrm{R}\left(\mathrm{S}_{\mathrm{i}}\right)$ 的前提完 成对层级的抽取, 根据可达集和先行集, 在 $\mathrm{i}=1$ 的时候, 这层的最高要素是 $\mathrm{S}_{1}$, 由此往下推, 获得最顶层的要素 集是 $\left\{\mathrm{S}_{3}\right\}$, 顺次获得第二层的要素是 $\left\{\mathrm{S}_{2}\right\}$, 第三层是 $\left\{\mathrm{S}_{1}\right\}$,

表 4 要素可达矩阵 $\mathbf{R}$

\begin{tabular}{|c|c|c|c|c|c|c|c|c|}
\hline & $\mathrm{S}_{1}$ & $\mathrm{~S}_{2}$ & $\mathrm{~S}_{3}$ & $\mathrm{~S}_{4}$ & $\mathrm{~S}_{5}$ & $\mathrm{~S}_{6}$ & $\mathrm{~S}_{7}$ & $\mathrm{~S}_{8}$ \\
\hline $\mathrm{S}_{1}$ & 1 & 1 & 1 & 0 & 0 & 0 & 0 & 0 \\
\hline $\mathrm{S}_{2}$ & 0 & 1 & 1 & 0 & 0 & 0 & 0 & 0 \\
\hline $\mathrm{S}_{3}$ & 0 & 0 & 1 & 0 & 0 & 0 & 0 & 0 \\
\hline $\mathrm{S}_{4}$ & 1 & 1 & 1 & 1 & 1 & 0 & 0 & 0 \\
\hline $\mathrm{S}_{5}$ & 1 & 1 & 1 & 1 & 1 & 0 & 0 & 0 \\
\hline $\mathrm{S}_{6}$ & 0 & 0 & 1 & 0 & 0 & 1 & 0 & 0 \\
\hline $\mathrm{S}_{7}$ & 1 & 1 & 1 & 1 & 1 & 0 & 1 & 1 \\
\hline $\mathrm{S}_{8}$ & 1 & 1 & 1 & 1 & 1 & 0 & 1 & 1 \\
\hline
\end{tabular}

\section{4 建立可达集与先行集}

可达集 $\mathrm{R}\left(\mathrm{S}_{\mathrm{i}}\right)$ ：就是可达矩阵里面要素 $\mathrm{Si}$ 所对应 的行内, 囊括着 1 的矩阵元素的对应的集合, 也就是所 有受要素 $S_{i}$ 影响的要素集; 先行集 $A\left(S_{i}\right)$ : 就是可达 矩阵里面要素 $\mathrm{S}_{\mathrm{i}}$ 所对应的列内, 囊括矩阵元素的集合, 其中 1 代表影响该特征的全部影响要素 $\mathrm{S}_{\mathrm{i}}$ 的要素集 ${ }^{{ }_{12} \mathbf{I}}$ 。 由可达矩阵, 可达集与先行集如表 5 。

\section{表 5 要素的先行集与可达集}

\begin{tabular}{|c|c|c|c|}
\hline $\begin{array}{l}\text { 要 } \\
\text { 素 }\end{array}$ & $\mathrm{R}(\mathrm{Si})$ & $\mathrm{Q}(\mathrm{Si})$ & $\begin{array}{c}\mathrm{R}(\mathrm{Si}) \cap \mathrm{Q} \\
(\mathrm{Si})\end{array}$ \\
\hline $\mathrm{S}_{1}$ & $\mathrm{~S}_{1}, \mathrm{~S}_{2}, \mathrm{~S}_{3}$ & $\mathrm{~S}_{1}, \mathrm{~S}_{4}, \mathrm{~S}_{5}, \mathrm{~S}_{7}, \mathrm{~S}_{8}$ & $\mathrm{~S}_{1}$ \\
\hline $\mathrm{S}_{2}$ & $\mathrm{~S}_{2}, \mathrm{~S}_{3}$ & $\begin{array}{c}\mathrm{S}_{1}, \mathrm{~S}_{2}, \mathrm{~S}_{4}, \mathrm{~S}_{5}, \mathrm{~S}_{7}, \\
\mathrm{~S}_{8}\end{array}$ & $\mathrm{~S}_{2}$ \\
\hline $\mathrm{S}_{3}$ & $\mathrm{~S}_{3}$ & $\begin{array}{c}\mathrm{S}_{1}, \mathrm{~S}_{2}, \mathrm{~S}_{3}, \mathrm{~S}_{4}, \mathrm{~S}_{5}, \\
\mathrm{~S}_{6}, \mathrm{~S}_{7}, \mathrm{~S}_{8} \\
\end{array}$ & $\mathrm{~S}_{3}$ \\
\hline $\mathrm{S}_{4}$ & $\begin{array}{c}\mathrm{S}_{1}, \mathrm{~S}_{2}, \mathrm{~S}_{3}, \\
\mathrm{~S}_{4}, \mathrm{~S}_{5}\end{array}$ & $\mathrm{~S}_{4}, \mathrm{~S}_{5}, \mathrm{~S}_{7}, \mathrm{~S}_{8}$ & $\mathrm{~S}_{4}, \mathrm{~S}_{5}$ \\
\hline $\mathrm{S}_{5}$ & $\begin{array}{c}\mathrm{S}_{1}, \mathrm{~S}_{2}, \mathrm{~S}_{3}, \\
\mathrm{~S}_{4}, \mathrm{~S}_{5}\end{array}$ & $\mathrm{~S}_{4}, \mathrm{~S}_{5}, \mathrm{~S}_{7}, \mathrm{~S}_{8}$ & $\mathrm{~S}_{4}, \mathrm{~S}_{5}$ \\
\hline $\mathrm{S}_{6}$ & $\mathrm{~S}_{3}, \mathrm{~S}_{6}$ & $\mathrm{~S}_{6}$ & $\mathrm{~S}_{6}$ \\
\hline $\mathrm{S}_{7}$ & $\begin{array}{c}\mathrm{S}_{1}, \mathrm{~S}_{2}, \mathrm{~S}_{3}, \\
\mathrm{~S}_{4}, \mathrm{~S}_{5}, \mathrm{~S}_{7} \\
\mathrm{~S}_{8} \\
\end{array}$ & $\mathrm{~S}_{7}, \mathrm{~S}_{8}$ & $\mathrm{~S}_{7}, \mathrm{~S}_{8}$ \\
\hline $\mathrm{S}_{8}$ & $\begin{array}{c}\mathrm{S}_{1}, \mathrm{~S}_{2}, \mathrm{~S}_{3}, \\
\mathrm{~S}_{4}, \mathrm{~S}_{5}, \mathrm{~S}_{7}, \\
\mathrm{~S}_{8}\end{array}$ & $\mathrm{~S}_{7}, \mathrm{~S}_{8}$ & $\mathrm{~S}_{7}, \mathrm{~S}_{8}$ \\
\hline
\end{tabular}

\section{5 划分要素层级}

根据此公式, 假如可达集 $\mathrm{R}\left(\mathrm{S}_{\mathrm{i}}\right)$ 以及先行集 $\mathrm{Q}\left(\mathrm{S}_{\mathrm{i}}\right)$ 的交集相同于可达集其本身, 那么证明此层级包括本元 素, 即: 若 $\mathrm{R}\left(\mathrm{S}_{\mathrm{i}}\right) \cap \mathrm{Q}\left(\mathrm{S}_{\mathrm{i}}\right)=\mathrm{R}\left(\mathrm{S}_{\mathrm{i}}\right)$, 则该层级的最
第四层是 $\left\{\mathrm{S}_{4} 、 \mathrm{~S}_{5}\right\}$, 底层是 $\left\{\mathrm{S}_{6} 、 \mathrm{~S}_{7} 、 \mathrm{~S}_{8}\right\}$, 见表 5 。

\section{6 建立解释结构模型并分析}

在级别分派结束后, 把各个要素从上至下按级别顺 序放置, 用有向线段表示其干系, 从而画出有向图。由 上述层级分析, 绘制影响长者食堂稳健运营因素的结构 解释模型, 如图 6 所示:

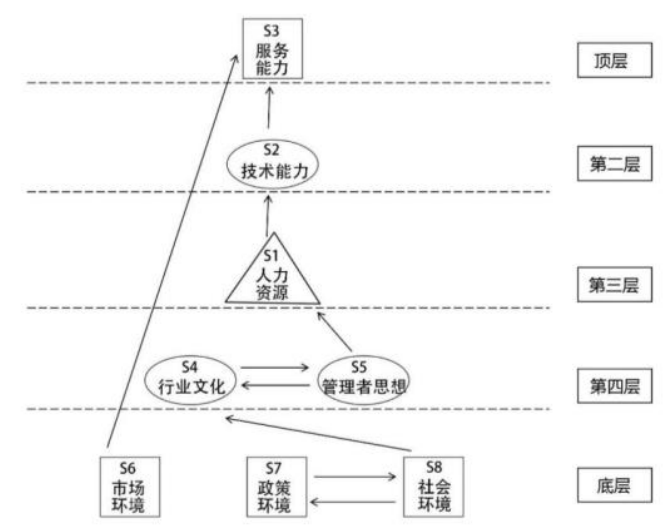

图 1 影响长者食堂稳健运营致因因素的解释结构模 型

由图 6 可得到影响长者食堂稳健运营关键因素以及 他们之间的相互作用关系:

1)最底层影响因子为市场环境（物价水平，也就是 场地租金、饭菜材料等成本)、政策环境（政府的条例 以及资金支持）、社会环境（社会捐助、企业扶持等）, 这三要素是其基本影响度, 是位于最底层的根源影响因 素。经营长者食堂需要支出的成本的波动变化会对食堂 的服务质量受到影响, 成本耗费更多, 在由于成本问题 而带来的盈利更少或亏损更多的情况下, 经营者需要调 整相应措施，对服务质量会有一定的影响; 政府的政策 和资金支持对社会的资助和企业的扶持起到互相推动 的作用，目前长者食堂的运营的资金来源基本是靠政府 的拨款以及社会各界的捐赠; 政策环境和社会环境的影 响对长者食堂的运营起到最基本的决定性作用; 同时, 社会环境中的企业共建或者扶持能为长者食堂提供更 完善的经营体系、经验等指导。

2) 第四层的元素为行业文化 (成熟的经营体系、宗 旨）和管理者思想先进程度（全心全意为老年人服务、 拥有经营的经验）。行业文化与管理者思想先进程度这 两个因素又相互影响，长者食堂成熟的经营体系、宗旨 等能对管理者思想或经营者有起到约束作用，管理者先 进的思想对长者食堂自身的行业文化起到促进完善的 作用。

3) 第三层的元素为人力资源, 是长者食堂内部的工 
作人员的人数、人员情况等, 此类因素对服务质量起到 一定程度的影响。

4) 第二层的元素为技术能力, 属于因素里的次级影 响因素。此处描述的技术能力也就是包括厨师对饭菜色 香味以及口感软硬程度的把握的能力。大部分长者的年 龄都比较大, 牙齿以及咬肌的功能都逐渐退化, 软质食 物更容易咀嚼以及下咽。除此以外, 服务的技术能力以 及各种问题的处置能力也有一定的影响力。

5) 位于解释结构模型顶层的因素是服务能力, 是表 层直接因素, 属于基础架构作用于承载体。在长者食堂 运营中, 服务质量, 食堂内部现有的服务类型是对食堂 的稳健运营产生直接影响, 可根据社区内需要被服务的 额长者数量配备足够的工作人员, 可以更好解决食堂饭 菜的搬运问题、收拾饭后残余以及清洁卫生, 为长者营 造更舒适的就餐环境, 便能够持续保持每天长者的就餐 人数, 达到长者食堂的初建目的; 除此以外, 工作人员 的素质程度如耐心满足不同长者的一些特殊需求等同 样是重要的, 可以让长者们倍感被关照的温暖。

\section{4.建议和总结}

长者食堂稳健运营的影响因素繁多, 同时各因素对 长者食堂运营的影响方式、规律和程度不尽相同, 其作 用机理也有差异。即便是同一种因素, 在不同的状态下 对长者食堂稳健运营的影响水平也可能有所差别。大都 情况下, 这些影响因素之间并非彼此独立存在的, 常常 存在着伴生、因果、转化等相关关系。为避免长者食堂 运营过程发生的各种风险, 使长者食堂更加稳健健康发 展, 为长者提供长久的养老服务, 对正确建设长者食堂 以及维持稳健运营的方向提出几点建议:

1) 政府应踊跃鼓动勉励企业、社会组织等介入长者 食堂的扶植或运营。许多长者食堂建设前存在场地的问 题, 不容易找到合适的显眼的位置、房屋租金比较贵, 尽然可利用闲置的国有产权房屋进行改造, 或者提供一 部分社区公共用房, 经改造后作为长者服务的场地。政 府在政策上可以发布相关法规对长者食堂的场地、经营 等建设补贴和奖励机制, 落实社区公共用房用于长者服 务, 方便企业拎包入驻提供服务。长者食堂的发展不能 仅靠政府的力量, 应当引入企业、社会慈善组织介入进 长者饭堂建设运营中。政府可以从制度建设、政策计划 上下功夫, 帮助企业乐于参加长者食堂建设项目, 并且 可以获得上、中、下游产业链协同可持续发展。

2) 给有困难的长者提供送餐到户的服务。老人的由 于年龄导致身体各方面机能下降, 行动不便、身体残疾 的老人占有大部分数量, 这些老人也是需要被关照的对 象。在老人无家人陪伴的情况下, 食堂可为老人提供送 餐上门服务, 根据老人的经济状况采取收取不同的费用 的措施 ${ }^{131}$ 。老人送餐服务可以为长者食堂赢得更多的口 碑以及用户, 同时也落实了居家养老, 增加老人幸福指 数。

3) 对长者食堂的服务体系、服务质量构成监督等机 制。结合 2020 国家养老政策, 政府加强托底保障, 加大
对基层养老服务设施建设投入力度, 建立健全长者食堂 服务体系和监督机制。长者食堂主要用户人群是老人群 体特别是弱势老人群体, 他们在个别些方面有特殊的需 求, 或者听力不好等身体上的问题, 因此要求长者食堂 的工作人员需要耐心和爱心, 为老人提供优质的服务。

4) 坚持兼顾社区老年人特殊需求。长者食堂的工作 人员需要考虑老年人这一群体的特殊性, 他们需要被者 的更软的食物, 方便咀嚼和下咽。抑或可针对部分老年 人的需求, 设计营养菜谱。进一步达到积极回应社会养 老需求, 将社区养老作为主要发展方向的目的 ${ }^{{ }^{1} 141}$ 。

总的来说, 目前我国社会养老服务体系正处于快速 发展阶段, 针对居家养老的社区服务设施建设尚处于萌 芽期 ${ }^{{ }^{1}}{ }^{151}$, 尚未确切完善, 基于国情, 借鉴先进地区发展 经验, 提早制定积极的应对策略, 居家养老体系的完善, 建立长者食堂类型体系, 也可以实施“抱团养老”的策略 $\mathbf{I}_{16}$ 。针对长者食堂在运营中的影响问题, 应积极应对和 解决, 不仅要政府、社会和企业的力量, 同时也需要各 个长者食堂自身内部服务体系的完善, 踊跃为居家养老 的社区老人提供优质的多元的服务, 为老人营造适合就 餐的空间环境, 确保落实居家养老、社区养老的模式 ${ }^{171}$ 。

\section{REFERENCES}

[1] Bai Lin. Research on the development of the old-age care service industry in Inner Mongolia from the perspective of effective supply [D]. Harbin University of Commerce, 2017.

[2] Wang Haitao, Fang Yu, Zhai Dehua, Wu Xiaolan, Luo Xiaohui, Qu Jiayao, Xin Tao. A new exploration of the oldage care model of urban core functional areas_-A survey based on 2400 samples of $\mathrm{X}$ urban district of Beijing [J]. Aging Science Research, 2013 , 1 (07): 62-71.

[3] Li Li. A Study on the Type System and Spatial Strategy of Community Elderly Canteens under the Home-based Pension Model_- Taking Guangzhou as an Example [J]. Art and Design (Theory), 2018, (12): 61-63

[4] Zuo Zhiping, Qi Zhenhong. Analysis of influencing factors of farmers 'green operation model_-Based on the survey of large-scale pig farmers in Hubei Province [J]. Jiangsu Agricultural Sciences, 2018, (16): 315-319.

[5] Yang Baolong. Operational Risk Analysis of Smart Community [D]. Dalian University, 2018.

[6] JOHN N WARFIELD. On arranging elements of a hierarchy in graphic from [J]. IEEE Transactions on Systems, Man, and Cybernetics, 1973, SMC-3 (2): 121-132

[7] Dong Peiwu, Liu Shiguo, Yang Mei, Wang Junmei. Research on Decision Model of Emergency Response of Matter Element Structure [J]. Systems Engineering and Electronic Technology, 2016, 38 (12): 2799-2806.

[8] Shi Liping Research on the Structure of Enterprise 
Emergency Ability Based on DEMATELISM [J]. Science and Technology Management Research, 2013 (5): 227-230

[9] Jiang Ning, Research on traffic safety emergency management system based on risk coupling [D]. Wuhan: Wuhan University of Technology, 2011

[10] Li Jun, Xie Shizhen, Gu Jianhua. ISM-based lowcarbon operation impact factor analysis and management model construction for small and medium-sized enterprises [J]. Ecological Economy, 2016, 32 (07): 74-77 + 105.

[11] Peng Jiao, Peng Xiaobing, Zhang Junjie. Research on the Influencing Factors of Rural Tourism Industrialization Development_—Based on Explanatory Structure Model [J]. Technology Economics and Management Research, 2019 (08): 124-128.

[12] Liao Hui. Analysis of the experience elements of railway mobile ticket purchase based on ISM model [J]. Science and Technology Innovation and Application, 2018 (11): $1-2+5$.

[13] Zhang Xiaoyu. Exploring the way of old-age care for the elderly in the city_-Taking Quanyechang Street in Tianjin as an example [D]. Tianjin Normal University, 2008.

[14] Li Xinhong. A comparative analysis of the performance of multi-level community pensions [D]. Nanchang University, 2016.

[15] Wei Tingting. Research on Comprehensive Evaluation of Home-based Pension Service in Urban Communities [D]. Zhengzhou University, 2017.

[16] Yang Jingzhong. Founding the Elders Canteen to promote the mode of holding elderly care services in groups [J] .CPPCC World, 2019 (05): 28-29.

[17] Xu Shoujiang, Luo Zhuqing. Research on community home-based pension information service [J]. Cooperative Economy and Technology, 2019 (18): 174-175. 